\title{
3D DESIGN OF CLOTHING IN MEDICAL APPLICATIONS
}

\section{Agnieszka Cichocka', Iwona Frydrych ${ }^{1}$, Małgorzata Zimniewska², Małgorzata Muzyczek², Przemysław Mikołajczak², Katarzyna Schmidt-Przewoźna², Barbara Romanowska², Mariola Pawlaczyk ${ }^{3}$, Izabella Krucińska ${ }^{4}$, Agnieszka Komisarczyk ${ }^{4}$, Stanisława Kowalska ${ }^{4}$, Lucyna Herczyńska ${ }^{4}$, Monika Urbaniak}

\author{
1 Lodz University of Technology, Institute of Textile Architecture, Poland \\ 2 Institute of Natural Fibres and Medical Plants, Poland \\ 3 Poznan University of Medical Science, Poland \\ 4 Department of Material and Commodity Sciences and Textiles Metrology, Lodz University of Technology, Poland \\ 5 Marko-Kolor Sp, Poland \\ Coresponding author. E-mail: agnieszka.cichocka@p.lodz.pl
}

\begin{abstract}
:
In this paper, we present a new methodology of clothing engineering construction for the medical application. Project "BIOAKOD" intends to propose clothing solutions for elderly people with dermatological defects. This purpose requires a particular concept of clothing because of the additional functionality expected in the clothing. It means that the proposed clothing needs to cover a human body in an aesthetic way and gives comfort of use it, and it should also bring an adding value in health treatment of people with dermatosis (psoriasis)..
\end{abstract}

\section{Keywords:}

$3 D$ fitting, personalization, clothing functionality, medical application

\section{Introduction}

Data from Eurostat (European Statistical Data Support) show the increase in the average age of European society. It is anticipated that the percentage of the population aged over 65 years compared with the entire European population will increase from $17.1 \%$ in 2008 to $30 \%$ in 2060 . It means that the increase in the number of elderly people in Europe during this period is about 67 million-from 84.6 million senior citizens in 2008 to 151.5 million senior citizens in 2060 . In the frame of BIOAKOD project, the new clothing model was designed for elderly women with some skin diseases and dysfunctions. Textile fabrics (woven or knitted) made of cotton and/or flax raw materials were coated by different kinds of herbs contained in the microcapsules. The development of microencapsulation technology has begun the development of cosmetotextiles used in clothing. The cosmetotextiles are usually the textile materials in which a part of clothing is incorporated with the microcapsules including substances, such as herbal extracts, compounds containing vitamin E, aloe vera, and others, to ensure the delivery of active matter with particular effect to the skin. The microcapsules under the special conditions release appropriate extracts of herbs, causing in this way adding the curing process. The aim of the presented problem in the "BIOAKOD" project was to create a personalized clothing, taking into account the morphology of a particular patient moving either alone or with dysfunction of body parts. The intention of this development is also to improve the quality of life of the elderly as well as of those requiring support in the treatment of skin and dermatological diseases.
The proposed approach to the problem was to ensure the correctness of the construction tailored to the specifics of the patient, and to propose a strategy for the implementation of clothing taking into account the technological process of manufacturing it in industrial conditions through the use of appropriate solutions.

\section{Experimental approach}

New technologies, such as 3D human body scanner and software for the virtual simulation of clothing, assuring the "fit" of clothing onto the mannequin in the virtual 3D space, can increase the efficiency and speed of clothing production process by the implementation of personalized patterns of clothing [1-3]. The 3D scanner is a tool accelerating receiving the anthropometric data of the human body. Using the scanner helps avoiding errors resulting from measurements done by different people and errors arising from accidentally written data or incorrect identification of anthropometric points. The scanning process also allows for measuring immediate and accurate dimension of the human body and for presenting the results of measurements numerically or volumetrically (3D volume)-the key point for the definition of clothing pattern models. Processing the scanned image using the appropriate technology allows for creating the numerical avatar of consumers' profiles and corresponding to their dimensions. It is done in order to follow the trend of buying clothes without leaving home and the idea of Mass Customization-using "virtual fitting clothing." Virtual simulation of clothing allows for disclosure of defects in the design, verification of drape and fit of the product to the silhouette of the virtual human body, and 
even the approval of correctness of virtually "sewn" clothing $[4,5]$. Software of this type allows generating parametric mannequins or entering the number of avatars created as a result of the scanning process. It is an interesting alternative for the people with disabilities, with specific anatomical defects or dysfunctions, for which the recommended and required is the so-called tailor-made clothing, which is designed to treat the skin disorders, especially for people who prefer to buy clothes via the Internet.

The design for this purpose clothing should be from one side tight to assure the contact of the skin with microcapsules and not too tight to assure the appropriate utility comfort of users.

Personalization process was a strategic for our project because of its multilevel application. On the one hand, it took the individual selection of the bioactive herbal extracts for encapsulation in microcapsules for each particular patient, to support the treatment of her dermatosis. On the other hand, the clothing design strongly depended on the location of the active elements in the clothing structure in relationship to the location of the skin lesions and adjustment of the clothing to the patient's body shape, taking into consideration individual style and color preferences.

Therefore, the 3D scanner was applied to personalize and facilitate the clothing design and construction process.

The plan of the research project to develop a system for designing the clothing tailored for the individual needs of patients is presented in Figure 1.

The presented strategy of special clothing creation process takes into account five elements. Collection of anthropometric data of users reflects the need to obtain a precise and quick description of the body of clothing wearer with the use of the scanner device. Due to the selected target of clothing wearers, some additional information about patients' habits and preferences were required.

\section{Methodology}

The adapted methodology is described in the research project on the implementation of personalized clothing for women, taking into account the morphology of silhouette of customer moving independently, with anatomical defects or dysfunctions of the body. The three of volunteers had the similar age, defining the seniors, and two of them were younger. The clothing structure design process has had a challenge to match each clothing size to the person (different size and body shape), also including the existing dysfunction of elderly women.

The proposed approach to the problem is to ensure the correctness of implementation of the structure matched to the specific patient, and it also aims in proposing a strategy for the implementation of clothes manufacturability taking into account the production process in the industrial conditions by the use of appropriate solutions. Twenty clothing sets for five volunteers, consisting of the upper and lower body clothing pieces (pants and blouses), were designed in accordance with the intended goal of trying to "replace" the traditional way of dosing the drug or medical supply for diseased areas of the skin. The clothing sets were designed for night and day outfits as the blouses and pants to ensure the continuous test of the healing effect of clothing in patients wearing the clothing.

The work included:

- A qualitative assessment of the patient's body (numeric avatar) and the development of qualitative recommendations for the implementation of clothing models.

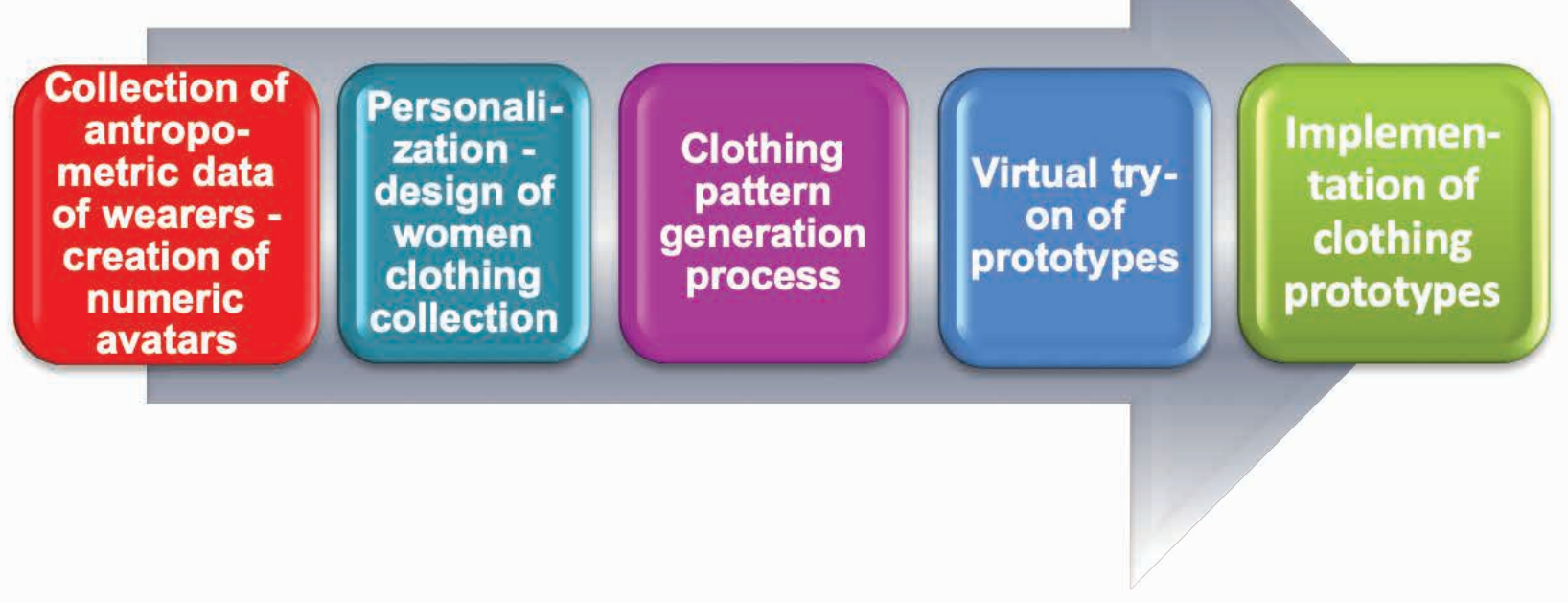

Figure 1. Concept of BIOAKOD clothing creation process. 
- Development of design modules of the BIOAKOD clothing collections, based on the specially selected dyestuffs for given materials and CAD system.

- Construction and modeling of standard clothing models for women's blouses and skirts according to the measurement and dermatosis of five female volunteers.

- Preparation of BIOAKOD clothing collections for the virtual sewing process and virtual clothing fitting for upper and lower body clothing pieces to avatars of volunteers for the day and night clothes.

- The implementation of clothing collections for the participating volunteers $1-5$.

In this work, the hardest aspect was the challenge to meet all the requirements of patient volunteers, concerning the style of clothing, preferences of materials, colors, sense of beauty, and comfort, which are difficult to attain [6-8]. The notion of beauty strictly depends on the choice of the individual. Moreover, the colors available for this project were obtained from dyestuffs extracted from very limited types of herbs that support curing particular dermatosis.

The potential of CAD system components in the field of modern technologies used for clothing design in our project presents a BIOAKOD design collection digitized using the KALEDO STYLE program. The numerical entry of the designer's idea allowed, in subsequent stages, to manage the whole process of designing and creating clothing more effectively, taking into account the design and material design phase. Every change introduced to this project independently, did not cause, at the same time, the need to regenerate the structure of modified projects. The use of such a CAD system solution causes those modifications; for example, modifications regarding the change of the color, the structure of the sleeves, fronts, and so on are available directly. This proves a significant acceleration and improvement in the entire process of preparing the implementation and production of clothing from the BIOAKOD collection, favorably affecting the time and costs of its implementation. All project "actors" have practically the knowledge available on-line, and thus they plan their next steps accordingly. This approach was in line with this project's assumption of the possibility of implementing the BIOAKOD collection in industrial conditions.

After evaluating the products drape on the body, the decision was taken (Figure 1) whether to continue the process of modeling and adaptation of models of clothes, until the volunteers receive a satisfactory effect of laying the clothing on the body. Successfully approved virtual fitting of a given clothing model meant the possibility of designing adequate layout templates as follows:

1. Verification of the structure of checking the drape models of clothes on the avatar corresponding to the dimensions of the morphological characteristics of the patient.

2. Applying the necessary adjustments and retouching the generated forms of clothing and drape a reassessment of the avatar.

3. Implementation of template layouts for a given product.

\section{Results and discussion}

In the BIOAKOD project, the personalization process was a very significant part of the process of clothing collection design. The challenge of this project was to propose to each patient the two suits: casual and for the night, which would be properly fitted to the given morphology and particular problem of dermatosis. Taking into account the number of selected wearers, varied by the age, morphology, as well as dermatosis aspect, 20 clothing models were prepared. It is worth to emphasize that each clothing model also takes into account the physical aspect of the wearer beauty seen as hair color, skin of face, and so on. Moreover, the palette of colors also defines the limits of
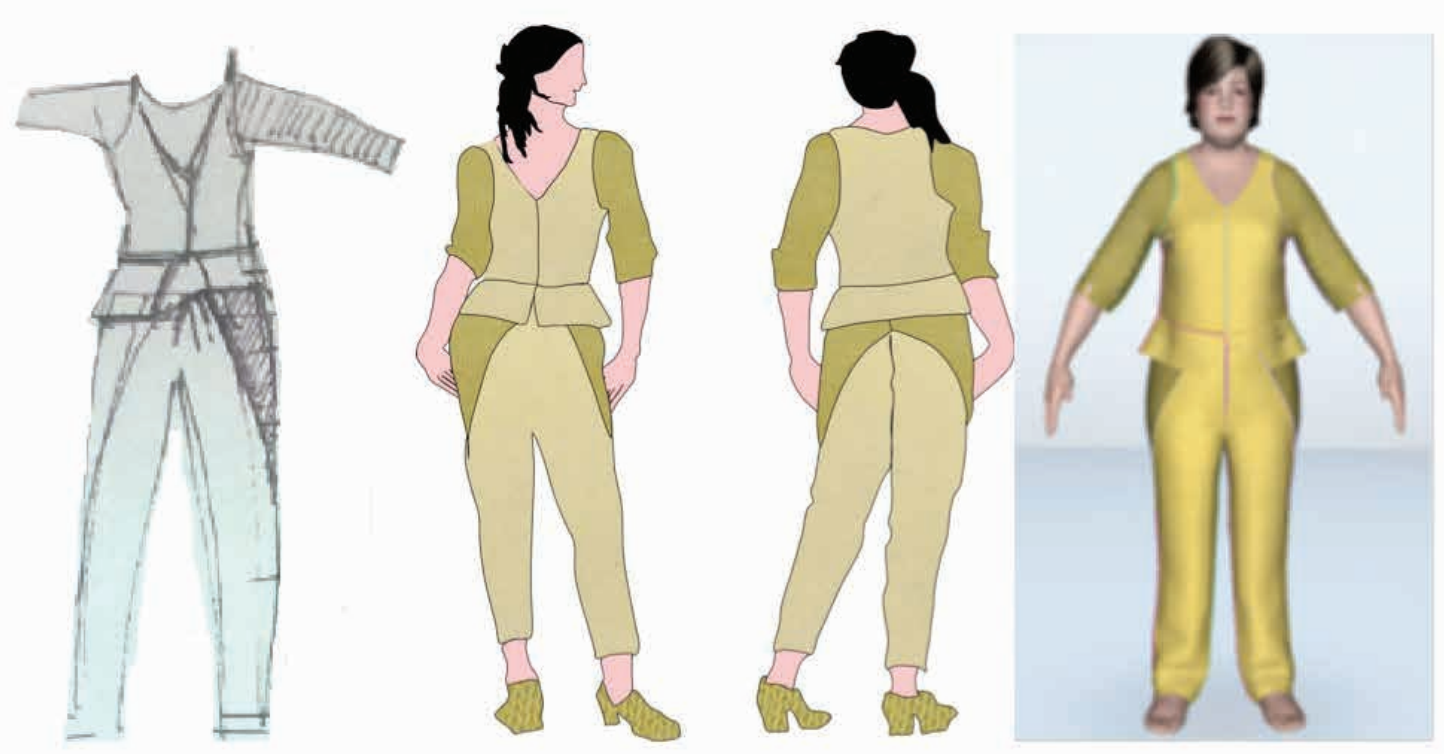

Figure 2. Example of designing and developing models of clothing prototypes. 
proposed clothing sets, because of used dyestuffs and chosen fabrics, where the idea of the ecological aspect managing the dyeing process with herbal plants was taken into account. The added value in clothing means their health aspect.

The use of such precise information obtained during the execution of anthropometric measurements of patients had a significant impact on designing "healthy" zones, particularly with regard to their location and size.

Generating the number of patient avatars were targeted to complement the information in the table of data which collected knowledge of behaviors and preferences of clothing for those ladies who were tested prototypes of clothing in this project. Virtual avatars were subjected to sequential modifications for parts localized as follows: legs, hands, arms, body height.

The example of process of designing and developing models of clothing prototypes is presented in Figure 2. The abovementioned process took place in three stages, where the use of 3D skills gave us the opportunity and advantage to personalize the clothing during the virtual try-on of BIOAKOD collection:

1. Construction of clothing standards, covering the upper and lower parts of the human body including the morphological characteristics of the user.

2. Iterative modeling procedure of standard models of trousers and blouses, in accordance with this project design. Interactive verification of clothing construction during the virtual simulation followed by necessary corrections of generated models of clothing (Figure 3).

3. Evaluation of the drape of casual and night outfits on the selected avatars and incorporating some corrections and re-evaluation of drape.

For the process of virtual fitting, the numerical "images" of the materials used in this project were applied. Digitization of materials from this project BIOAKOD was preceded by using the physical parameters of materials obtained from the KES-F system and also by including the visualization of clothing appearance based on more realistic outlooks because of numerical views of used materials. Optimizing the criteria of the selection of modeled clothing such as the ease allowance as well as the material stresses, offered by software, was carried out (Figure 4). Moreover, this tool also helped to assume that the dimensions of clothing and pattern construction process with healing elements were designed in a properly way. Due to $3 \mathrm{D}$ interactive simulation of clothing, the alteration of the clothing patterns could be still possible.

The implementation of pattern markers was carried out using the Lectra CAD with system module called DIAMINO EXPERT. The previously created clothing models of BIOKAOD collection were numerically processed, which resulted in the data ready to be exported in the production model (in our case, systems printed on the paper). Paper pattern sets included, among others, width of material used to obtain pattern markers, method of material placement, and parameters ensuring proper course of the cutting process, including cutting method or cutting machine type, for example, stationary cutting machine, laser cutting head.

The five stages (Figure 1) taking into account the virtual tryon of prototypes were essential for the next stage of prototype creation process, that is, the clinical tests on five human beings wearing and evaluating BIOAKOD clothing functionality.

\section{Conclusions}

Implementation of new clothing technologies to the preparation of clothing collection, which helps to cure skin diseases, can be assured by an application process of sewing without difficulties. Moreover, the data generated in this way can be transferred easily to the manufacturer and enable saving time and costs.

Simulations of clothing products using the clothing forms obtained in 3D and traditional, which are part of the BIOAKOD collection, show coherence in the process of preparing clothing implementation.
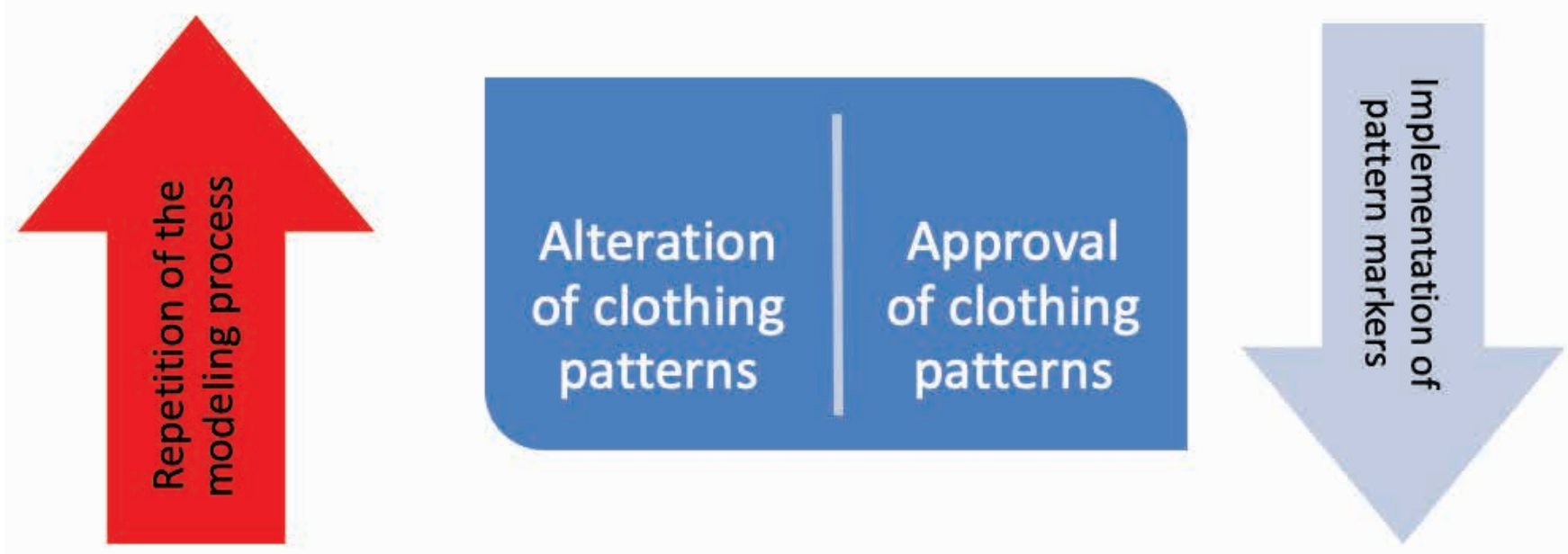

Figure 3. Decision-making procedure to terminate or continue the process of pattern alteration. 


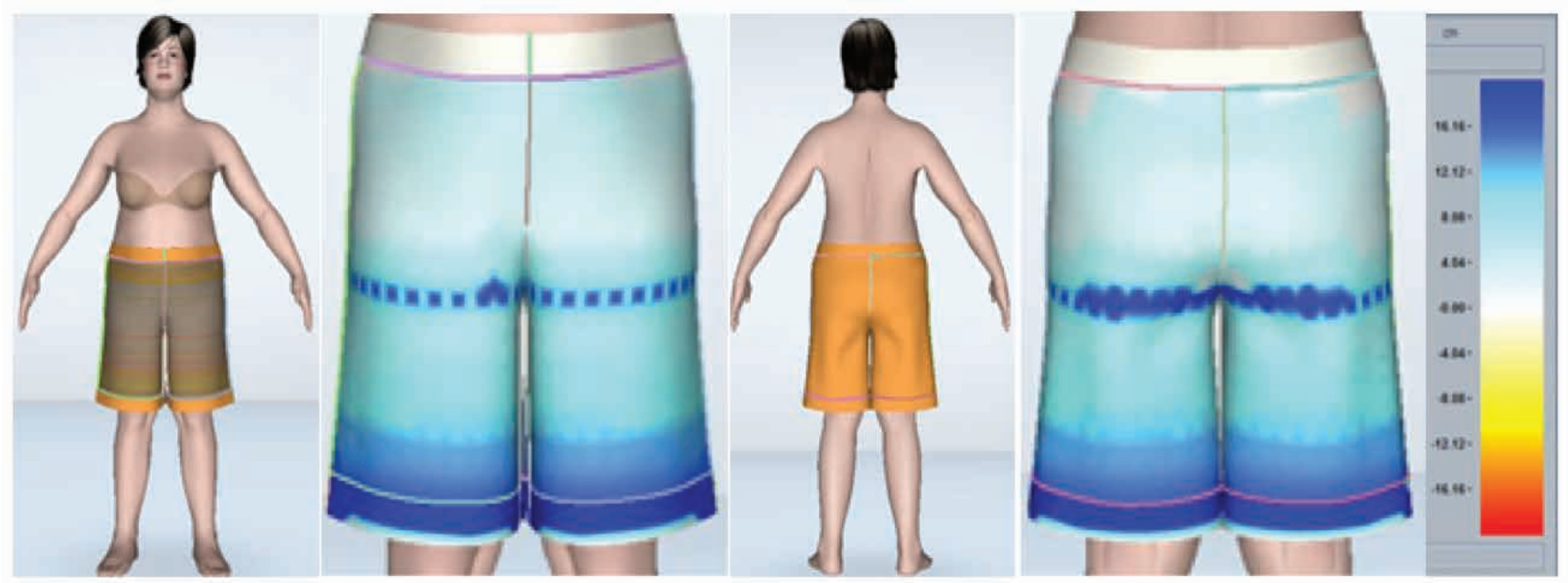

Figure 4. BIOAKOD clothing virtual try-on of the selected prototypes.

The implementation of clothing patterns of the BIOAKOD collection, made on the basis of model drawings and approved during virtual clothing simulations (3D forms), served to support the clothing for positively tested clinical conditions. This study has been completed with the filing of a patent submission (no. $P$ 411869), titled "The clothing acting as a dressing supplement in the therapy of dermatological diseases."

The products developed in this project will provide a new BIOAKOD quality of clothing in the market-a support intended for the people suffering from dermatological diseases, for disabilities, and for seniors. Moreover, it is expected that in 2020 about $25 \%$ of the European population will be accounted for the elderly and disabled [9]. This raises the necessity of conducting research in which $3 \mathrm{D}$ technology streamlines the process of generating clothing for people with difficult silhouette and for disabled persons $[10,11]$.

\section{Acknowledgment}

This work was partially supported by Structural Founds in the frame of the project titled "Bioactive clothing with healing care properties." BIOAKOD, financed by Applied Research Programme of the National Centre for Research and Development in Poland (PBS 1-Project no. 177463, program path A), was studied at the Lodz University of Technology.

\section{References}

[1] Cichocka, A., Bruniaux, P., Koncar, V. (2007). Modelling of virtual garment design in $3 D$. Research Journal of Textile and Apparel, 11(4), 55-63.

[2] Cichocka, A., Bruniaux, P., Frydrych, I. (2014). 3D garment modelling - creation of a virtual mannequin of the human body. Fibres \& Textiles in Eastern Europe, 22(6), 123-131.

[3] Cichocka, A., Bruniaux, P., Frydrych, I. (2016). 3D garment modelling - conception of its structure in $3 D$. Fibres \& Textiles in Eastern Europe, 24(3), 121-128.

[4] Mengna, G., Victor, E., Kuzmichev, D. C. (2015). Adolphe Human-friendly design of virtual system "female bodydress". Autex Research Journal, 15(1), 19-29.

[5] Bruniaux, P., Cichocka, A., Frydrych, I. (2016). 3D digital methods of clothing creation for disabled people. Fibres \& Textiles in Eastern Europe, 5(119), 125-131.

[6] Central Research and Development Center Clothing Industry. (1983). Construction standards of women's clothing (in Polish), Poland,

[7] Lewandowska, E. (1992). Design and modeling of women's clothing (in Polish). Oświatowiec (Toruń, Poland).

[8] Von Jutta, J., Rüdiger, C. (1994). Systemschnitt I, II, (in German). Schiele \& Schön Bücher (Berlin, Germany).

[9] BIOAKOD. Web site: http://bioakod.pl/.

[10] Surikova, O. V., Kuzmichev, V., Surikova, G. I. (2017). Improvement of clothes fit for different female bodies. Autex Research Journal, 17(2), 111-119.

[11] Yan, H., Pascal, B., Xianyi, Z., Kaixuan, L., Antonela, C., Yan, C. (2018). Visual-simulation-based personalized garment block design method for physically disabled people with scoliosis (PDPS). Autex Research Journal, 18(1), 35-45. 